\title{
Erratum to: This is Our Brain
}

\section{Erratum to:}

\section{J. Hendrikse, This is Our Brain,}

DOI 10.1007/978-981-10-4148-8

In the original version of the book, the newly received correction to change the layout has to be incorporated. The erratum book has been updated with the change.

The updated original online version of this book can be found at DOI 10.1007/978-981-10-4148-8 